\title{
Overexpression of melanopsin in the retina restores visual function in Royal College of Surgeons rats
}

\author{
MINGMING LIU, JIAMAN DAI, WENYI LIU, CHONGJIAN ZHAO and ZHENG QIN YIN \\ Southwest Hospital/Southwest Eye Hospital, Third Military Medical University, Chongqing 400038, P.R. China
}

Received December 11, 2014; Accepted October 1, 2015

DOI: $10.3892 / \mathrm{mmr} .2015 .4549$

\begin{abstract}
Retinitis pigmentosa (RP) is a pathological condition leading to progressive visual decline resulting from continual loss of photoreceptor cells and outer nuclear layers of the retina. The aim of the present study was to explore whether melanopsin was able to restore retinal function and inhibit its degeneration by acting in a similar manner to channel rhodopsins. Royal College of Surgeons rats, which were used as an animal model of inherited retinal degeneration, were subjected to sub-retinal injection with melanopsin overexpression vector (AV-OPN4-GFP). Immunohistochemical and western blot analyses were used to detect the distribution and protein expression of melanopsin in the retina, revealing that melanopsin was gradually reduced with increasing age of the rats, which was due to loss of dendritic axons of intrinsically photosensitive retinal ganglion cells. Animals injected into both eyes were subjected to a behavioral open-field test, revealing that melanopsin overexpression reduced the loss of light sensitivity of the rats. In a flash electroretinography experiment, the b-wave and response to light flash stimuli at three and five weeks following injection with AV-OPN4-GFP were higher compared to those in eyes injected with AV-GFP $(\mathrm{P}<0.05)$. In conclusion, the present study showed that during retinal degeneration, the expression of melanopsin was significantly decreased, while vector-mediated overexpression of melanopsin delayed the loss of visual function in rats.
\end{abstract}

\section{Introduction}

Retinitis pigmentosa (RP) is an pathological condition leading to progressive visual decline resulting from continual loss of photoreceptor cells and outer nuclear layers of the retina (1). At present, no cures for this degenerative retinal disease are

Correspondence to: Dr Zheng Qin Yin, Southwest Hospital/Southwest Eye Hospital, Third Military Medical University, 30 Gaotanyan Street, Chongqing 400038, P.R. China

E-mail: qinz.yin@yahoo.com

Key words: Royal College of Surgeons rats, melanopsin, sub-retinal injection, adenovirus - opsin 4 - green fluorescence protein, flash electroretinogram available. Experimental approaches for the development of treatments of RP have advanced in recent years, which include drug treatments $(2,3)$, stem-cell transplantation (4), gene therapy (5) and light sensitivity-modulating proteins (6).

Treatment approaches using light sensitivity-enhancing proteins such as channel rhodopsins (ChR) and melanopsin have recently emerged as a hotspot of RP research. ChR2, which was first described in green algae, is a light-gated ion channel which evokes ultrafast membrane depolarization and mediates an action potential $(7,8)$. Of note, ChR2 has been demonstrated to be able to restore the function of degenerative retinal diseases. Unlike ChR2, the photopigment melanopsin is expressed by intrinsically photosensitive retinal ganglion cells (ipRGC) in the human retina (9). Melanopsins mediate a broad range of physiological responses, including circadian rhythm, modulation of sleep and alertness (10-12). The light-evoked response mediated by melanopsin is slower but more durable than that of ChR2 in the absence of photoreceptor-based input (12). However, whether melanopsin has the potential to restore visual function similarly to ChR2 has remained elusive. Therefore, the present study evaluated the effects of melanopsin in Royal College of Surgeons (RCS) rats, which are widely used as a model of inherited retinal degeneration. These animals carry a mutation in the Mertk gene expressed in retinal pigment epithelial cells, which results in progressive photoreceptor cell death and loss of electrophysiological response $(13,14)$. The present study aimed to determine whether sub-retinal injection of melanopsin-overexpressing adenovirus may restore visual function in RCS rats in order to explore the feasibility of using melanopsin as a novel therapeutic for retinal degenerative diseases.

\section{Materials and methods}

Experimental animals. A total of 18 healthy neonatal RCS rats (4-week-old) were provided by the Experimental Animal Center of Southwest Hospital, Third Military Medical University (Chongqing, China) of mixed gender. Of these rats, 10 were used for behavioral testing and 8 were used for flash electroretinography (FERG). Prior to the start of the experiments, the rats were acclimatized for 1 week. The rats were reared in a light-controlled room with a 12-h light/dark cycle at $22-25^{\circ} \mathrm{C}$ and $50-60 \%$ humidity. All experimental protocols were in accordance with the Guidance Suggestions for the Care and Use of Laboratory Animals, formulated by the Ministry of 
Science and Technology of the People's Republic of China (15), and approved by the ethics committee of the Third Military Medical University (Chongqing, China). Experiments were performed in the laboratory of Southwest Eye Hospital from August 2012 to December 2013.

Viral vectors. Two viral vectors were used: Adenovirus-opsin4-greenfluorescent protein(AV-Opn4-GFP) and the empty vector AV-GFP. Full-length mouse melanopsin (GenBank accession no. 6693702) was cloned into pAV vector under the transcriptional control of the murine cytomegalovirus promoter. These two constructs were packaged at the GeneChem Biological Company virus production core (Shanghai, China). The packaged viruses were concentrated and dissolved in phosphate-buffered saline (PBS) to 1.08 or $2.2 \times 10^{13}$ genome copies per milliliter for AV-Opn4-GFP or AV-GFP, respectively.

Sub-retinal injection of adenovirus. For anesthesia, animals at the age of five weeks were subjected to intramuscular thigh injection with a mixture of $10 \mathrm{mg} / \mathrm{kg}$ ketamine (Sigma-Aldrich, St. Louis, MO, USA) and $1 \mathrm{mg} / \mathrm{kg}$ xylazine (Sigma-Aldrich). Oxybuprocaine 0.4\% (Santen Pharmaceutical Co.,Ltd., Osaka, Japan) eye drops were used for superficial anesthesia. Under aseptic conditions, the RCS rats were individually moved to an animal operating table under a microscope on a biological super-clean bench. $2 \mu 1$ viral suspension was injected into the sub-retinal space in the superior hemisphere of the eyes. In the group subjected to behavioral testing, five rats received AV-Opn4-GFP injection into both eyes in the experimental group, while the control group consisted of five untreated RCS rats. In the group subjected to FERG testing, in order to avoid individual differences, the right eye of the rats received AV-Opn4-GFP injection, while the left eye was injected with a control viral vector (AV-GFP; $\mathrm{n}=8$ ).

Immunohistochemistry. At 3, 5 and 7 weeks following injection with the vectors, the eyes were immediately enucleated from RCS rats sacrificed by decapitation under deep anesthesia via intramuscular injection of $20 \mathrm{mg} / \mathrm{kg}$ ketamine and $2 \mathrm{mg} / \mathrm{kg}$ xylazine. For immunohistochemical analysis, the whole-mount retina was isolated and blocked with 5\% bovine serum albumin (Beyotime Institute of Biotechnology, Haimen, China) diluted in $10 \%$ goat serum (Beyotime Institute of Biotechnology) in PBS for $1 \mathrm{~h}$ at room temperature $\left(25^{\circ} \mathrm{C}\right)$. The blocked retina was washed with PBS for $5 \mathrm{~min}$ for times. Next, the retina was incubated with rabbit anti-mouse anti-melanopsin polyclonal antibody (1:500; cat. no. ab19306; Abcam, Cambridge) overnight at $4^{\circ} \mathrm{C}$ and washed in PBS three times for $15 \mathrm{~min}$ each. Subsequently, the retinas were incubated with $\mathrm{Cy} 3$-conjugated goat anti-rabbit immunoglobulin G (1:500; cat. no. A0516; Beyotime Institute of Biotechnology) for $4 \mathrm{~h}$ at room temperature and washed three times in PBS for $15 \mathrm{~min}$ each. A fluorescence microscope (Zeiss LSM 510, Carl Zeiss AG, Oberkochen, Germany) was used to capture images. The number of ipRGCs was counted by fluorescence microscopy under x200 magnification. For each sample, nine fields of view with the same size $(200 \times 300 \mu \mathrm{m})$ were randomly selected. The length of the axons was also measured under a fluorescence microscope (x400 magnification) and 27 cells were randomly selected $(16,17)$.

Western blot analysis. The retina was isolated using the liquid nitrogen method (17) and lysed using a cell lysis solution [50 mM Tris-HCl buffer (pH 7.4), $150 \mathrm{mM} \mathrm{NaCl}, 1 \%$ Triton X-100, $1 \%$ sodium deoxycholate, $0.1 \%$ SDS, sodium orthovanadate, sodium fluoride, EDTA and leupeptin; P0013B; Beyotime Institute of Biotechnology]. The cell lysates were separated by $12 \%$ SDS-PAGE and electrotransferred onto nitrocellulose (NC) membranes (Whatman, Fairfield, CT, USA). After blocking with $5 \%$ de-fatted milk in PBS containing $0.05 \%$ Tween- 20 overnight at $4^{\circ} \mathrm{C}$, the membranes were incubated with the anti-melanopsin antibody overnight at $4^{\circ} \mathrm{C}$. After washing, all membranes were incubated with rat polyclonal GAPDH antibody (1:1,000; cat. no. CW0101; CWBio, Beijing, China) for $1 \mathrm{~h}$ at room temperature and horseradish peroxidase-conjugated goat anti-rabbit $\operatorname{IgG}(1: 2,000$; cat. no. A0208; Beyotime Institute of Biotechnology) at room temperature for $1 \mathrm{~h}$. The $\mathrm{NC}$ membranes were visualized using an enhanced chemiluminescence kit (Applied Biosystems; Thermo Fisher Scientific, Waltham, MA, USA) and scanned using the Odyssey infrared imaging system with the Odyssey Application software (V1.2.15; LI-COR Biosciences, Lincoln, NE, USA). Quantification of melanopsin was performed by densitometric analysis of the protein bands $(16,17)$.

Open field test. The open field test box was $45 \times 30 \times 40 \mathrm{~cm}$ in size and divided into a white open field and a dark zone with a door $(10 \times 10 \mathrm{~cm})$ between them. To test rats in the open field ( 5 weeks post-injection), the rats were placed in the dark zone for $2 \mathrm{~min}$ for adaptation and the door was then opened to observe the behavior of the rats. The time spent in the dark zone and white open field was recorded. The open field test was performed under 300 lux light intensity and recorded using a video camera to enable subsequent evaluation. The bottom surface of the box was cleaned with $70 \%$ ethanol prior to testing of each animal $(18,19)$.

FERG recording. FERG recording was performed on animals at 3,5 and 7 weeks following injection with melanopsin-overexpression/empty vector. Animals were allowed to adapt to darkness for almost $12 \mathrm{~h}$ and prepared for recording under dim red light. After anesthesia, pupils were dilated with tropicamide and phenylephrine eye drops (Santen Pharmaceutical Co., Ltd). FERG responses of both eyes were recorded simultaneously using goldwire loops (Roland Consult, Brandenburg a.d. Havel Germany). The cornea was frequently treated with $0.9 \%$ saline to prevent its de-hydration and allow electrical contact with the recording electrode. Two needle electrodes were inserted under the skin of the angulus oculi temporal to serve as reference electrodes, while the other electrodes were placed in the tail to serve as the grounding electrodes. The b-waves were acquired using the Reti-scan system (Roland Consult, Brandenburg a.d. Havel, Germany). Dark-adapted intensity responses to stimuli with intensities of $-0.5,-0.02,0.5$ and $1 \mathrm{Log}\left(\mathrm{cd} \mathrm{sec} / \mathrm{m}^{2}\right)$ were measured. To avoid any adapting effect of the previous flash, the flash interval was set as $60-120 \mathrm{sec}$ depending on stimulus intensity. 
A

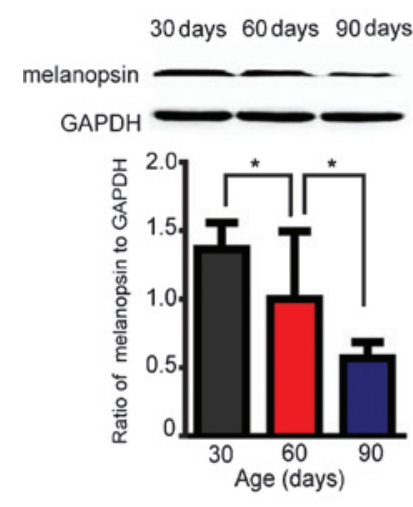

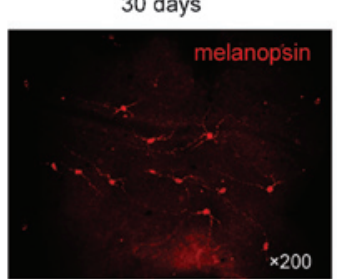

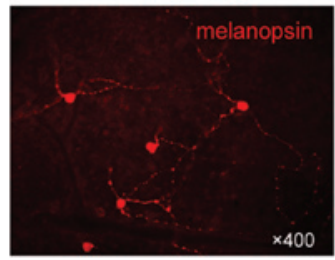

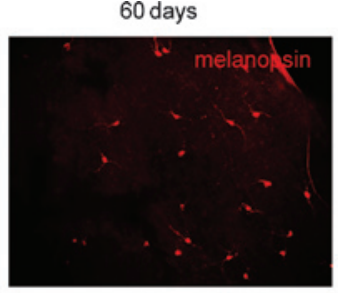

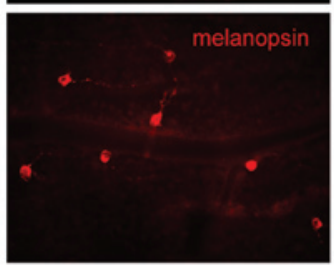

90 days

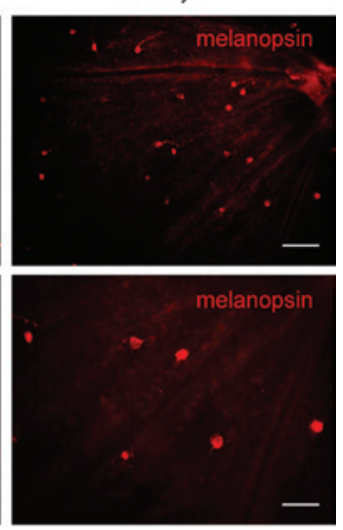

C

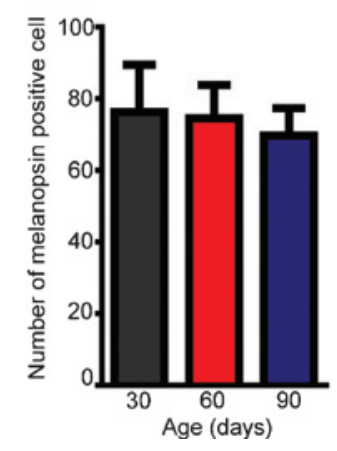

D

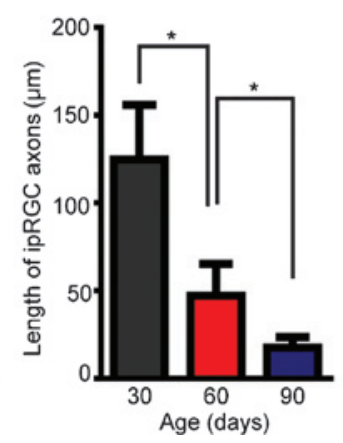

Figure 1. Distribution of ipRGC in rat retinas. (A) Western blot analysis of melanopsin expression. (B) Immunofluorescence assay for melanopsin-positive cells (red) and length of ipRGC axons in rat retinas (magnification, x200 in top row and x400 in bottom row). Quantification of (C) melanopsin-positive cells and (D) length of ipRGC axons. Values are expressed as the mean \pm standard deviation. ${ }^{*} \mathrm{P}<0.05$. ipRGC, intrinsically photosensitive retinal ganglion cells.

Statistical analysis. Data analysis was performed using the SPSS 13.0 statistical package (SPSS, Inc., Chicago, IL, USA). Values are expressed as the mean \pm standard deviation. Data were analyzed using the independent-samples $t$ test to compare the differences between two treatment modalities/groups in the FERG and behavioral experiments. $\mathrm{P}<0.05$ was considered to indicate a statistically significant difference between values.

\section{Results}

Melanopsin expression is decreased during retinal degradation due to loss of dendritic axons. Western blot analysis demonstrated that in the retinal degeneration model, melanopsin expression levels in the retinas of rats aged 30,60 and 90 days decreased with increasing age (Fig. 1A). However, during this time, the number of melanopsin-positive cells did not decrease, as indicated by immunohistochemistry (Fig. 1B and C). Assessment of the average axon length of ipRGCs revealed significant shortening after 60 and 90 days compared with axon length at 30 postnatal days (Fig. 1D). These results confirmed that in RCS rats as a model of chronic retinal degeneration, melanopsin expression is gradually decreased with increasing age. This diminished expression of melanopsin was demonstrated to result from the decreased length of dendritic axons of ipRGCs, but not the loss of the number of ipRGCs.

Vector-mediated overexpression of melanopsin. The retinas of rats at 3,5 and 7 weeks following injection of AV-OPN4-GFP (at the age of 8, 10 and 12 weeks, respectively) were subjected to western blot analysis of melanopsin. The results confirmed that at 3 and 5 weeks following sub-retinal injection of AV-OPN4-GFP, a significant overexpression of melanopsin was achieved in the retina, compared with its expression in the control vector-injected eyes $(\mathrm{P}<0.05)$ (Fig. 2).

Melanopsin overexpression in retina reduces loss of light sensitivity in RCS rats. Previous studies have demonstrated that normal rats avoid open, bright spaces, and that this innate tendency can be utilized as the basis of a simple test of their ability to detect light $(18,19)$. In the open-field test, rats are placed in an illuminated open field with access to a dark zone. The time spent by the rats in the open space is recorded using a camera. The total time of the test is $300 \mathrm{sec}$. The results showed that the RCS rats at 10 weeks of age injected with the empty vector AV-GFP did not possess a sufficient number of photoreceptors to mediate light avoidance, resulting in $217.8 \pm 24.13 \mathrm{sec}(\mathrm{n}=5)$ in the dark zone, whereas RCS rats injected with AV-OPN4-GFP spent 292.4 $\pm 5.59 \mathrm{sec}(\mathrm{n}=5)$ in the dark zone $(\mathrm{P}<0.05)$ (Fig. 3). This result indicated that melanopsin overexpression in the retina inhibited the loss of light sensitivity in a rat model of ongoing retinal degradation.

Melanopsin enhances the retinal $b$-wave and response to flash light stimuli. FERG is a means of measuring the entire function of the retina in response to stimulation with flashed light. It has been previously reported that the b-wave amplitude of RCS rats decreases gradually and is almost completely 
A

Melanopsin GAPDH

B

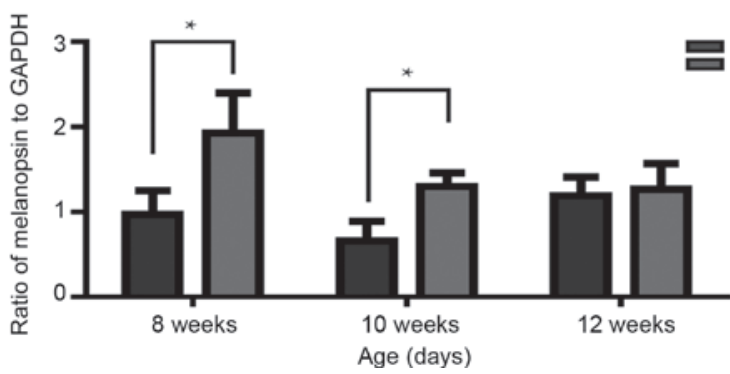

Figure 2. Protein expression of melanopsin in the sub-retinal injected eye at 8,10 and 12 weeks of age (equivalent to 3,5 and 7 weeks after vector injection). (A) Representative western blots for melanopsin expression. GAPDH was used as a loading control. (B) Melanopsin expression was quantified by desitometric analysis. Values are expressed as the mean \pm standard deviation. ${ }^{*} \mathrm{P}<0.05$. AV-OPN4-GFP, adenovirus - opsin 4 - green fluorescence protein; Con, control.

A

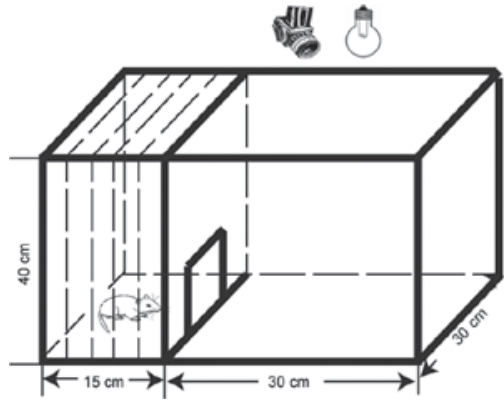

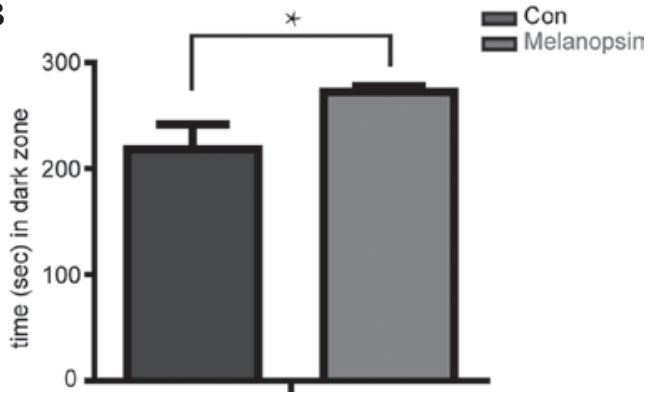

Figure 3. Open-field test. (A) Schematic diagram of the set-up of the open-field test. The cage was divided into a white open field (right) and a dark zone (left) connected by a door $(10 \times 10 \mathrm{~cm})$, through which the rats could pass freely. (B) Time spent in the dark zone in the two groups of rats. Values are expressed as the mean \pm standard deviation. ${ }^{*} \mathrm{P}<0.05$. Con, control.

A

Inj-3W (age, 8 weeks)
Inj-5W (age, 10 weeks)

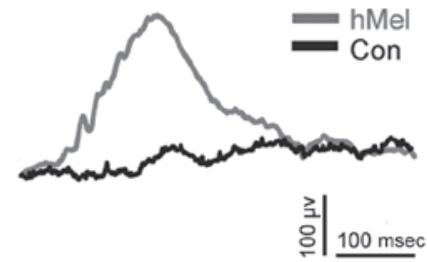

Inj-5W (age, 10 weeks) b-wave

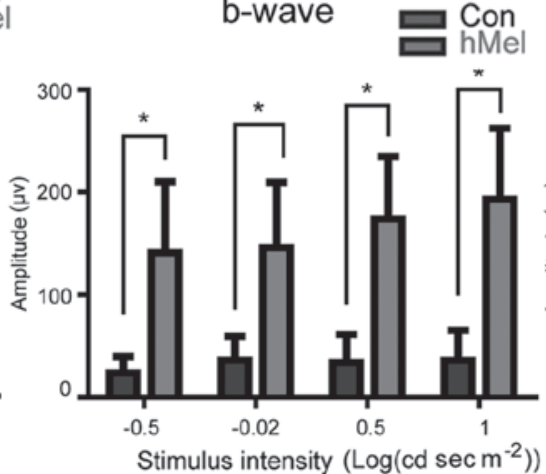

Inj-7W (age, 12 weeks)

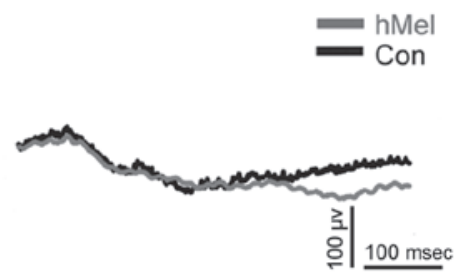

Inj-7W (age, 12 weeks) b-wave
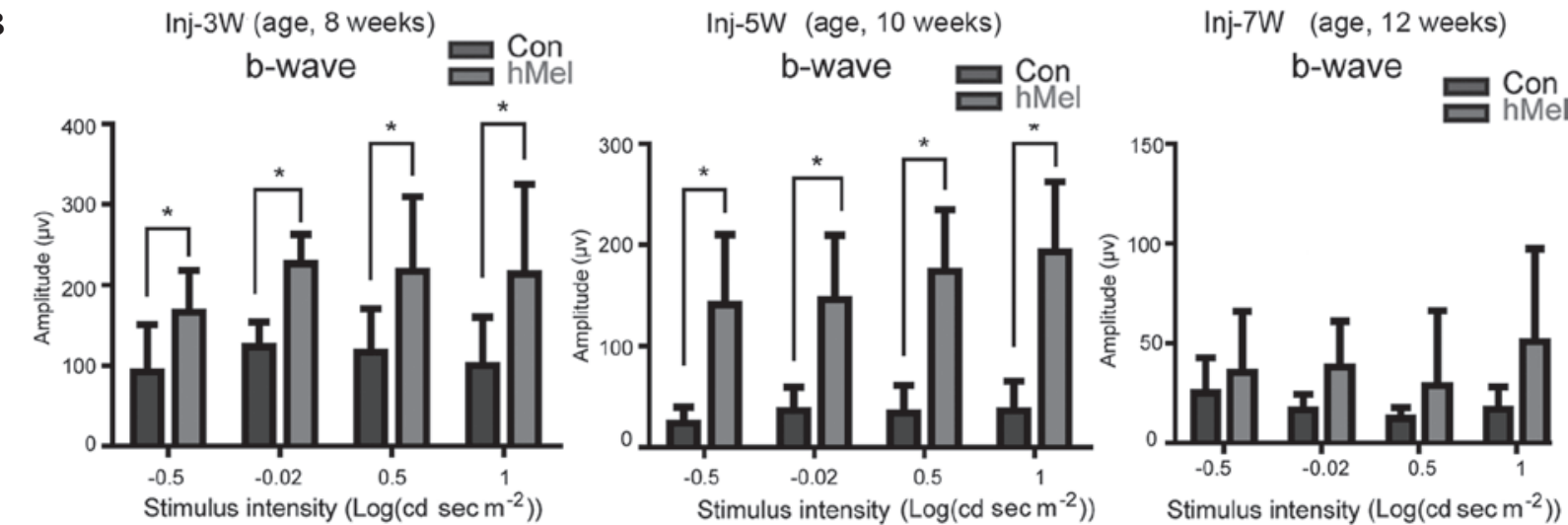

Figure 4. Flash electroretinogram response of rats at 8,10 and 12 weeks of age (3,5 and 7 weeks after sub-retinal injection). (A) Representative traces of FERG at 3,5 and 7 weeks after sub-retinal injection. The grey line indicates the right (treated) eye and the black line represents the left (control) eye. (B) Mean amplitude of the b-wave at different stimulus intensities 3,5 and 7 weeks after sub-retinal injection. Data are presented as the mean \pm standard deviation (n=8). The grey indicates the right (treated) eye and the black represents the left (control) eye. Values are expressed as the mean \pm standard deviation ("P<0.05). Con, control; hMel; injected with melanopsin expression vector; Inj-3W, 3 weeks post-injection. 
abolished at 60 days of age (20). In the present study, the b-wave amplitude at 10 weeks of age was reduced compared with that at 8 weeks of age, and was completely abolished at 12 weeks of age in the rat eyes injected with empty vector (Fig. 4A). However, the eyes of RCS rats which had received sub-retinal injections of AV-OPN4-GFP at the age of five weeks showed an enhanced b-wave amplitude compared with that of empty vector-injected eyes at 8 and 10 weeks of age $(n=8)$ (Fig. 4A). However, at 12 weeks, the amplitude of the $b$-waves in the melanopsin-overexpressing eyes could not be restored. Similarly, the response to stimuli with dosed light flashes at various intensities was decreased in the empty vector-injected eyes at weeks 10 and 12 of age compared with that at 8 weeks. In the eyes injected with AV-OPN4-GFP, the response was significantly increased compared with that of empty vector-injected eyes at 8 and 10 weeks of age $(\mathrm{P}<0.05)$; however, differences were not significant at 12 weeks of age (P>0.05) (Fig. 4B).

\section{Discussion}

The structure of the mammalian retina is highly organized and all light detection is performed by two visual pathways: The image-forming and the non-image-forming systems (21). In the traditional model, the retina contains two types of photoreceptor - the rod and the cone. These perform the first step of the visual process by capturing light and transducing it into electrical signals. However, in recent years, a novel type of specialized retinal ganglion cell, namely the ipRGC, was discovered, which expresses the photopigment melanopsin and is intrinsically photosensitive $(21,22)$. Upon their discovery, ipRGCs were first considered to be involved in the circadian rhythm, and within the last few years it has become evident that these photoreceptors mediate light detection to regulate behavioral and physiological responses to light $(23,24)$. It has now been suggested that the ipRGC family has up to five distinct cell types (termed M1-5) with various morphological characteristics and locations of their dendritic arbors (25). M1 ipRGCs are well established as predominantly being responsible for circadian photoentrainment and the pupillary light reflex $(26,27)$. The non-M1 ipRGCs may be responsible for image-forming behaviors via projection to the lateral geniculate nucleus (28). In addition, a study on bipolar cells showed that bipolar axons communicate with outer stratifying melanopsin cells in the retina of primates (29).

FERG is a widely used method for detecting the entire function of the retina. With the aggravation of retinal degeneration, RCS rats are depleted of almost all of their rods and cones, and in spite of a few surviving cells, retinal function is almost completely lost, followed by the complete abolishment of the electrophysiological response (20). In the present study, western blot and immunofluorescence analysis were used to assess melanopsin expression during retinal degeneration, revealing that its protein expression was gradually decreased, particularly at 60 postnatal days. The length of retinal axons was significantly shortened after 30 postnatal days. A viral vector was used to deliver melanopsin to the retina in order to supplement the reduced melanopsin levels with the aggravation of retinal degeneration; this led to a delay in the time-dependent loss of the b-wave response. Of note, these findings indicated a role for melanopsin and ipRGCs in the image-forming systems of the eye.

Behavioral studies have shown that normal rat avoids open, bright spaces, while the rats with retinal degeneration spend a decreased amount of time in a dark area $(18,19,30)$. Of note, in the present study RCS rats sub-retinally injected with AV-OPN4-GFP spent a longer duration in the dark zone compared with untreated RCS rats. This indicated that melanopsin may be able to delay retinal degradation or enhance the efficiency of the remaining photoreceptors in the retina, leading to an increased electrical signal and light sensitivity. In addition, the FERG assay showed that melanopsin rescued the electrophysiological response to the flashed light, which was transmitted to the image-forming system. Therefore, modulation of melanopsin expression may be a novel therapeutic method for treating patients with retinal degeneration. However, the life time of the AV used to deliver melanopsin into the sub-retinal region was relatively low, as in the present study, its effects were diminished at 7 weeks following injection in terms of protein expression of melanopsin and the b-wave response. Thus, a further study by our group will use adeno-associated virus instead of AV for the delivery of melanopsin, which may be more suitable for use in vivo. Future studies should be performed to enhance the current understanding of the molecular mechanisms of action of the endogenous opsin melanopsin, including regulation of the electrophysiological response to flash light and the effects of its modulation on visual performance.

\section{Acknowledgements}

The authors would like to thank Professor Yaochen Li (Shantou University Medical College, Shantou, China) for his advice on the design of the present study. This work was supported by a grant from the Science Foundation of Chongqing, Major International (Regional) Joint Research Project (no. CSTC2013GJH210004 to ZY).

\section{References}

1. Sung CH, Davenport CM, Hennessey JC, Maumenee IH, Jacobson SG, Heckenlively JR, Nowakowski R, Fishman G, Gouras $\mathrm{P}$ and Nathans $\mathrm{J}$ : Rhodopsin mutations in autosomal dominant retinitis pigmentosa. Proc Natl Acad Sci USA 88: 6481-6485, 1991.

2. Rayapudi S, Schwartz SG, Wang X and Chavis P: Vitamin A and fish oils for retinitis pigmentosa. Cochrane Database Syst Rev 12: CD008428, 2013.

3. Zarbin MA, Arlow T and Ritch R: Regenerative nanomedicine for vision restoration. Mayo Clin Proc 88: 1480-1490, 2013.

4. Kamao H, Mandai M, Okamoto S, Sakai N, Suga A, Sugita S, Kiryu J and Takahashi M: Characterization of human induced pluripotent stem cell-derived retinal pigment epithelium cell sheets aiming for clinical application. Stem Cell Reports 2: 205-218, 2014.

5. Jiang L, Frederick JM and Baehr W: RNA interference gene therapy in dominant retinitis pigmentosa and cone-rod dystrophy mouse models caused by GCAP1 mutations. Front Mol Neurosci 7: 25, 2014.

6. Pan ZH, Ganjawala TH, Lu Q, Ivanova E and Zhang Z: ChR2 Mutants at L132 and T159 with improved operational light sensitivity for vision restoration. PLoS One 9: e98924, 2014.

7. Nagel G, Szellas T, Huhn W, Kateriya S, Adeishvili N, Berthold P, Ollig D, Hegemann P and Bamberg E: Channelrhodopsin-2, a directly light-gated cation-selective membrane channel. Proc Natl Acad Sci USA 100: 13940-13945, 2013. 
8. Bi A, Cui J, Ma YP, Olshevskaya E, Pu M, Dizhoor AM and Pan ZH: Ectopic expression of a microbial-type rhodopsin restores visual responses in mice with photoreceptor degeneration. Neuron 50: 23-33, 2006

9. Hattar S, Liao HW, Takao M, Berson DM and Yau KW: Melanopsin-containing retinal ganglion cells: Architecture, projections, and intrinsic photosensitivity. Science 295: 1065-1070, 2002.

10. Ramsey DJ, Ramsey KM and Vavvas DG: Genetic advances in ophthalmology: The role of melanopsin-expressing, intrinsically photosensitive retinal ganglion cells in the circadian organization of the visual system. Semin Ophthalmol 28: 406-421, 2013.

11. Roecklein KA, Wong PM, Miller MA, Donofry SD, Kamarck ML and Brainard GC: Melanopsin, photosensitive ganglion cells and seasonal affective disorder. Neurosci Biobehav Rev 37: 229-239, 2013.

12. Koizumi A, Tanaka KF and Yamanaka A: The manipulation of neural and cellular activities by ectopic expression of melanopsin. Neurosci Res 75: 3-5, 2013.

13. Kyger M, Worley A and Adamus G: Autoimmune responses against photoreceptor antigens during retinal degeneration and their role in macrophage recruitment into retinas of RCS rats. J Neuroimmunol 254: 91-100, 2013.

14. McGill TJ, Prusky GT, Douglas RM, Yasumura D, Matthes MT, Lowe RJ, Duncan JL, Yang H, Ahern K, Daniello KM, et al: Discordant anatomical, electrophysiological and visual behavioral profiles of retinal degeneration in rat models of retinal degenerative disease. Invest Ophthalmol Vis Sci 53: 6232-6244, 2012.

15. The Ministry of Science and Technology of the People's Republic of China. Guidance suggestions for the care and use of laboratory animals. Beijing, China, 2006.

16. Tao Z, Dai J, He J, Li C, Li Y and Yin ZQ: The influence of $\mathrm{NaIO}(3)$-induced retinal degeneration on intra-retinal layer and the changes of expression profile/morphology of DA-ACs and mRGCS. Mol Neurobiol 47: 241-260, 2013.

17. Li YC, Li CS, Chen ZS, He JR, Tao Z and Yin ZQ: A MicroRNA, mir133b, suppresses melanopsin expression mediated by failure dopaminergic amacrine cells in RCS rats. Cell Signal 24: 685-698, 2012

18. Go RE, Hwang KA, Kim SH, Lee MY, Kim CW, Jeon SY, Kim YB and Choi KC: Effects of anti-obesity drugs, phentermine and mahuang, on the behavioral patterns in Sprague-Dawley rat model. Lab Anim Res 30: 73-78, 2014.
19. Lin B, Koizumi A, Tanaka N, Panda S and Masland RH: Restoration of visual function in retinal degeneration mice by ectopic expression of melanopsin. Proc Natl Acad Sci USA 105: 16009-16014, 2008.

20. Pinilla I, Lund RD and Sauvé Y: Contribution of rod and cone pathways to the dark-adapted electroretinogram (ERG) b-wave following retinal degeneration in RCS rats. Vision Res 44: 2467-2474, 2004.

21. Münch M and Kawasaki A: Intrinsically photosensitive retinal ganglion cells: Classification, function and clinical implications. Curr Opin Neurol 26: 45-51, 2013

22. Pickard GE and Sollars PJ: Intrinsically photosensitive retinal ganglion cells. Rev Physiol Biochem Pharmacol 162: 59-90, 2012.

23. Ramsey DJ, Ramsey KM and Vavvas DG: Genetic advances in ophthalmology: The role of melanopsin-expressing, intrinsically photosensitive retinal ganglion cells in the circadian organization of the visual system. Semin Ophthalmol 28: 406-421, 2013.

24. Semo M, Gias C, Ahmado A and Vugler A: A role for the ciliary marginal zone in the melanopsin-dependent intrinsic pupillary light reflex. Exp Eye Res 119: 8-18, 2014.

25. Hu C, Hill DD and Wong KY: Intrinsic physiological properties of the five types of mouse ganglion-cell photoreceptors. JNeurophysiol 109: 1876-1889, 2013.

26. Güler AD, Ecker JL, Lall GS, Haq S, Altimus CM, Liao HW, Barnard AR, Cahill H, Badea TC, Zhao H, et al: Melanopsin cells are the principal conduits for rod-cone input to non-image-forming vision. Nature 453: 102-105, 2008.

27. Gamlin PD, McDougal DH, Pokorny J, Smith VC, Yau KW and Dacey DM: Human and macaque pupil responses driven by melanopsin-containing retinal ganglion cells. Vision Res 47 : 946-954, 2007.

28. Ecker JL, Dumitrescu ON, Wong KY, Alam NM, Chen SK, LeGates T, Renna JM, Prusky GT, Berson DM and Hattar S: Melanopsin-expressing retinal ganglion-cell photoreceptors: Cellular diversity and role in pattern vision. Neuron 67: 49-60, 2010.

29. Grünert U, Jusuf PR, Lee SC and Nguyen DT: Bipolar input to melanopsin containing ganglion cells in primate retina. Vis Neurosci 28: 39-50, 2011.

30. Padilla E, Shumake J, Barrett DW, Holmes G, Sheridan EC and Gonzalez-Lima F: Novelty-evoked activity in open field predicts susceptibility to helpless behavior. Physiol Behav 101: 746-754, 2010. 